Supplement of Hydrol. Earth Syst. Sci., 19, 3073-3091, 2015

http://www.hydrol-earth-syst-sci.net/19/3073/2015/

doi:10.5194/hess-19-3073-2015-supplement

(C) Author(s) 2015. CC Attribution 3.0 License.

(c) (1)

Supplement of

\title{
Water savings potentials of irrigation systems: global simulation of processes and linkages
}

\section{J. Jägermeyr et al.}

Correspondence to: J. Jägermeyr (jonas.jaegermeyr@ pik-potsdam.de)

The copyright of individual parts of the supplement might differ from the CC-BY 3.0 licence. 


\section{Development of a new input for spatially explicit distribution of irrigation systems}

The extent of irrigated areas (both equipped and actually irrigated) is relatively well documented and has been disaggregated to grid cell level by Stefan Siebert and Petra Döll et al. (S. Siebert et al., 2005, 2010; S Siebert et al., 2014; Stefan Siebert, Döll, Feick, Hoogeveen, \& Frenken, 2007) which is included in the MIRCA2000 land use data set (Portmann et al., 2010) that we employ throughout our study (area equipped for irrigation). Information on the irrigation system that is in place is however less well documented. The FAO Aquastat data base (FAO, 2014) provides national irrigated areas separated for the three irrigation systems surface, sprinkler and drip (area equipped for irrigation). But such areas are not consistently available for all countries and significant gaps and errors are apparent. The International Commission on Irrigation and Drainage (ICID) also provides estimates on national shares of sprinkler and drip irrigated areas (ICID, 2012), but unfortunately not for all countries. Rohwer et al. (2007) also compiled a country statistic on the share of irrigation systems, based on FAO, ICID and other sources but it is not comprehensive for all countries.

Here we compile a new country-level database on the extent of the three main irrigation systems surface, sprinkler and drip, based on FAO (2014), ICID (2012) and Rohwer et al. (2007). In general, the total extent of actually irrigated areas is relatively consistent between MIRCA2000, AQUASTAT and ICID, but estimates of the extent of each system are often inconsistent or missing. We assume that the areas on which the three systems are implemented sum up to total area equipped for irrigation (i.e. that there are no other systems). Our primary data source is FAO (2014) and in case of missing or inconsistent data we fill in with data from ICID (2012) or Rohwer et al. (2007). In countries where only the total irrigated area is available, we allocate shares according to neighboring countries. The complete list is shown in Table A1.

Furthermore, we disaggregate country shares of each irrigation system to the irrigated area from MIRCA2000. For this, we developed and employed decision rules to decide which irrigation system is most suited for a CFT. These decisions are based on Brouwer et al. (1988), Sauer et al. (2010) and Fischer (2012) and are summerized in the suitability table (Tab. 2 in main paper). The basic rationale is that drip irrigation is most suitable for high value crops, and sprinkler irrigation is suitable for most row, field and tree crops (Brouwer et al., 1988). From the 12 CFTs in LPJmL we only excluded rice (always surface) and tropical roots (not irrigated at all) from this decision rule.

Our algorithm works in that first in each country all cells are selected that have areas with dripsuitable CFTs. From that pool, we randomly sample CFT fractions until the target area for drip systems is fulfilled in each country. This procedure is repeated 1000 times and we employ the iteration for which the target area is met most precisely. Then, sprinkler systems are attributed accordingly and the remaining free cell fraction for irrigation is assigned to surface irrigation. In the end, each CFT in each cell is assigned to one of the three irrigation systems.

The extent of irrigated areas by CFT changes each year (S Siebert et al., 2015). Irrigation system distributions that were assigned for the previous year are kept in place for the next year, if the extent of irrigated cropland does not decline. On newly added cropland irrigation systems are distributed according to the rules above. 
Table S1: Country-level extent ${ }^{\mathrm{a}}$ and shares of surface, sprinkler and drip irrigated areas, compiled from FAO (2014), ICID (2012) and Rohwer et al. (2007).

\begin{tabular}{|c|c|c|c|c|c|c|c|c|c|c|c|}
\hline Country & $\begin{array}{l}\text { Equipped } \\
\text { for surface } \\
\text { irrigation }\end{array}$ & $\begin{array}{l}\text { Equipped for } \\
\text { sprinkler } \\
\text { irrigation }\end{array}$ & $\begin{array}{l}\text { Equipped } \\
\text { for drip/ } \\
\text { localized }\end{array}$ & Sum & $\begin{array}{l}\text { Total area } \\
\text { equipped } \\
\text { (Aquastat) }\end{array}$ & $\begin{array}{l}\text { Total area } \\
\text { equipped } \\
\text { (ICID) }\end{array}$ & \begin{tabular}{|l|} 
Actually \\
irrigated, \\
\% (FAO)
\end{tabular} & $\begin{array}{l}\text { Surface } \\
\text { Share }\end{array}$ & $\begin{array}{l}\text { Sprinkler } \\
\text { Share }\end{array}$ & $\begin{array}{l}\text { Drip/localized } \\
\text { Share }\end{array}$ & Notes \\
\hline Afghanistan & $3,094.00$ & 114.00 & 0.00 & $3,208.00$ & $3,208.00$ & & 53.99 & 0.964 & 0.036 & 0.000 & added 3094 to surf to meet FAO total area \\
\hline Albania & 397.00 & 0.03 & 0.00 & 397.03 & 188.40 & & 56.53 & 1.000 & 0.000 & 0.000 & added 473.4 to surf to meet FAO total area \\
\hline Algeria & 473.40 & 40.00 & 0.00 & 513.40 & 513.40 & & 88.29 & 0.922 & 0.078 & 0.000 & \\
\hline Andorra & 0.00 & 0.00 & 0.00 & 0.00 & 0.00 & & & 0.000 & 0.000 & 0.000 & \\
\hline Angola & 85.53 & 0.00 & 0.00 & 85.53 & 85.53 & & 13.47 & 1.000 & 0.000 & 0.000 & \\
\hline Antigua and Barbuda & 0.00 & 0.03 & 0.10 & 0.13 & 0.13 & & & 0.023 & 0.192 & 0.785 & added 85 to surf to meet FAO total area \\
\hline Argentina & $1,949.00$ & 281.00 & 127.00 & $2,357.00$ & $2,357.00$ & & 91.73 & 0.827 & 0.119 & 0.054 & \\
\hline Armenia & 247.50 & 25.00 & 1.00 & 273.50 & 273.50 & & 64.35 & 0.905 & 0.091 & 0.004 & \\
\hline Australia & $1,831.00$ & 524.00 & 191.00 & $2,546.00$ & $2,546.00$ & $2,545.00$ & & 0.719 & 0.206 & 0.075 & \\
\hline Austria & 0.00 & 115.00 & 2.00 & 117.00 & 117.00 & & 37.13 & 0.000 & 0.983 & 0.017 & added 115 to sprink to meet FAO total area \\
\hline Azerbaijan & 817.90 & 607.00 & 0.10 & $1,425.00$ & $1,425.00$ & $1,433.00$ & 95.30 & 0.574 & 0.426 & 0.000 & \\
\hline Bahamas, The & 0.00 & 0.00 & 0.00 & 0.00 & 0.00 & & 0.00 & 0.000 & 0.000 & 0.000 & \\
\hline Bahrain & 3.39 & 0.16 & 0.47 & 4.02 & 4.02 & & 100.00 & 0.844 & 0.040 & 0.116 & \\
\hline Bangladesh & $5,050.00$ & 0.00 & 0.00 & $5,050.00$ & $5,050.00$ & & 88.32 & 1.000 & 0.000 & 0.000 & \\
\hline Barbados & & & & 0.00 & 5.44 & & & 0.000 & 0.000 & 0.000 & \\
\hline Byelarus & 0.00 & 131.00 & 0.00 & 131.00 & 115.00 & & & 0.000 & 1.000 & 0.000 & \\
\hline Belgium & 0.00 & 23.35 & 0.00 & 23.35 & 23.35 & & 24.33 & 0.000 & 1.000 & 0.000 & added 23 to sprink to meet FAO total area \\
\hline Belize & 3.20 & 0.30 & 0.05 & 3.55 & 3.55 & & 100.00 & 0.901 & 0.085 & 0.014 & Shares based on neighboring countries \\
\hline Benin & 5.04 & 4.57 & 1.36 & 10.97 & 23.04 & & 74.65 & 0.460 & 0.416 & 0.124 & \\
\hline Bhutan & 27.68 & 0.00 & 0.00 & 27.68 & 31.91 & & 100.00 & 1.000 & 0.000 & 0.000 & \\
\hline Bolivia & 275.90 & 17.60 & 3.70 & 297.20 & 297.20 & & 100.00 & 0.928 & 0.059 & 0.012 & \\
\hline $\begin{array}{l}\text { Bosnia and } \\
\text { Herzegovina }\end{array}$ & 1.40 & 1.40 & 0.20 & 3.00 & 3.00 & & & 0.467 & 0.467 & 0.067 & Shares based on neighboring countries \\
\hline Botswana & 0.22 & 0.89 & 0.27 & 1.38 & 1.44 & & 100.00 & 0.158 & 0.646 & 0.196 & \\
\hline Brazil & $2,619.00$ & $2,446.00$ & 334.80 & $5,399.80$ & $5,400.00$ & $4,450.00$ & 96.81 & 0.485 & 0.453 & 0.062 & \\
\hline
\end{tabular}




\begin{tabular}{|c|c|c|c|c|c|c|c|c|c|c|c|}
\hline Brunei & 0.59 & 0.35 & 0.06 & 1.00 & 1.00 & & 63.00 & 0.589 & 0.350 & 0.061 & Based on Rohwer et al. (2007) \\
\hline Bulgaria & 80.60 & 21.00 & 3.00 & 104.60 & 104.60 & 588.00 & 69.45 & 0.771 & 0.201 & 0.029 & \\
\hline Burkina Faso & 25.39 & 3.90 & 0.44 & 29.73 & 29.73 & & 85.00 & 0.854 & 0.131 & 0.015 & \\
\hline Burundi & 6.96 & 0.00 & 0.00 & 6.96 & 6.96 & & 64.08 & 1.000 & 0.000 & 0.000 & \\
\hline Cambodia & 269.50 & 0.00 & 0.00 & 269.50 & 353.60 & & 89.71 & 1.000 & 0.000 & 0.000 & \\
\hline Cameroon & 17.02 & 5.43 & 0.00 & 22.45 & 22.45 & & 100.00 & 0.758 & 0.242 & 0.000 & \\
\hline Canada & 180.90 & 683.00 & 6.03 & 869.93 & 869.90 & 870.00 & 100.00 & 0.208 & 0.785 & 0.007 & \\
\hline Cape Verde & 0.00 & 0.00 & 0.20 & 0.20 & 3.48 & & 65.50 & 0.000 & 0.000 & 1.000 & \\
\hline $\begin{array}{l}\text { Central African } \\
\text { Republic }\end{array}$ & 0.00 & 0.00 & 0.00 & 0.00 & 0.14 & & 51.11 & 0.000 & 0.000 & 0.000 & \\
\hline Chad & 26.52 & 3.75 & 0.00 & 30.27 & 30.27 & & 86.55 & 0.876 & 0.124 & 0.000 & \\
\hline Chile & 801.30 & 57.40 & 249.80 & $1,108.50$ & $1,109.00$ & $1,090.00$ & 98.65 & 0.723 & 0.052 & 0.225 & \\
\hline China & $59,338.00$ & $2,841.00$ & 759.50 & $\begin{array}{l}62,938.5 \\
0\end{array}$ & $62,938.00$ & $59,300.00$ & 86.15 & 0.943 & 0.045 & 0.012 & \\
\hline Colombia & 856.80 & 36.90 & 6.30 & 900.00 & $1,087.00$ & & 36.25 & 0.952 & 0.041 & 0.007 & Based on Rohwer et al. (2007) \\
\hline Comoros & 0.00 & 0.00 & 0.00 & 0.00 & 0.13 & & 65.38 & 0.000 & 0.000 & 0.000 & \\
\hline Congo-Brazzaville & 0.22 & 0.00 & 0.00 & 0.22 & 0.22 & & 100.00 & 0.995 & 0.000 & 0.005 & \\
\hline Cook Islands & 0.00 & 0.00 & 0.00 & 0.00 & 0.00 & & & 0.000 & 0.000 & 0.000 & \\
\hline Costa Rica & 86.28 & 10.15 & 5.08 & 101.51 & 101.50 & & 100.00 & 0.850 & 0.100 & 0.050 & \\
\hline Ivory Coast & 11.75 & 36.00 & 0.00 & 47.75 & 47.75 & & & 0.246 & 0.754 & 0.000 & \\
\hline Croatia & 1.84 & 1.67 & 0.12 & 3.63 & 3.63 & & & 0.506 & 0.462 & 0.032 & \\
\hline Cuba & 366.60 & 402.70 & 19.49 & 788.79 & 788.80 & & & 0.465 & 0.511 & 0.025 & \\
\hline Cyprus & 1.98 & 1.98 & 35.59 & 39.54 & 39.54 & & & 0.050 & 0.050 & 0.900 & \\
\hline Czech Republic & 22.53 & 11.00 & 5.00 & 38.53 & 38.53 & 153.00 & 51.67 & 0.585 & 0.285 & 0.130 & \\
\hline North Korea & $1,460.00$ & 0.00 & 0.00 & $1,460.00$ & $1,460.00$ & & 92.53 & 1.000 & 0.000 & 0.000 & \\
\hline $\begin{array}{l}\text { DR Congo, former } \\
\text { Zaire }\end{array}$ & 10.00 & 0.00 & 0.00 & 10.00 & 10.00 & & & 1.000 & 0.000 & 0.000 & \\
\hline Denmark & 0.00 & 391.50 & 43.50 & 435.00 & 435.40 & & 58.36 & 0.000 & 0.900 & 0.100 & Based on Rohwer et al. (2007) \\
\hline Djibouti & 0.00 & 0.00 & 0.00 & 0.00 & 1.01 & & 38.34 & 0.000 & 0.000 & 0.000 & \\
\hline Dominica & 0.00 & 0.00 & 0.00 & 0.00 & 0.00 & & & 0.000 & 0.000 & 0.000 & \\
\hline Dominican Republic & 269.70 & 0.00 & 0.00 & 269.70 & 306.50 & & 100.00 & 1.000 & 0.000 & 0.000 & \\
\hline
\end{tabular}




\begin{tabular}{|c|c|c|c|c|c|c|c|c|c|c|c|}
\hline Ecuador & 663.90 & 170.10 & 19.40 & 853.40 & $1,500.00$ & & 62.80 & 0.778 & 0.199 & 0.023 & \\
\hline Egypt & $3,029.00$ & 171.90 & 221.40 & $3,422.30$ & $3,422.00$ & $3,420.00$ & 100.00 & 0.885 & 0.050 & 0.065 & \\
\hline El Salvador & 41.56 & 2.49 & 1.18 & 45.22 & 45.23 & & 74.82 & 0.919 & 0.055 & 0.026 & \\
\hline Equatorial Guinea & 0.00 & 0.00 & 0.00 & 0.00 & 0.00 & & & 0.000 & 0.000 & 0.000 & \\
\hline Eritrea & 4.10 & 0.00 & 0.00 & 4.10 & 4.10 & & 100.00 & 1.000 & 0.000 & 0.000 & \\
\hline Estonia & 0.00 & 3.68 & 0.00 & 3.68 & 0.46 & 1.00 & 71.18 & 0.000 & 1.000 & 0.000 & \\
\hline Ethiopia & 283.20 & 6.36 & 0.01 & 289.57 & 289.60 & & 100.00 & 0.978 & 0.022 & 0.000 & \\
\hline Faroe Islands & 0.00 & 0.00 & 0.00 & 0.00 & 0.00 & & & 0.000 & 0.000 & 0.000 & \\
\hline Fiji & 3.00 & 0.00 & 0.00 & 3.00 & 3.00 & & & 1.000 & 0.000 & 0.000 & Shares based on neighboring countries \\
\hline Finland & 0.00 & 58.78 & 9.80 & 68.58 & 68.58 & 70.00 & 21.90 & 0.000 & 0.857 & 0.143 & \\
\hline France & $1,416.90$ & $1,379.80$ & 103.30 & $2,900.00$ & $2,642.00$ & $2,900.00$ & 57.23 & 0.489 & 0.476 & 0.036 & $\begin{array}{l}\text { Based on ICID (2012) and Rohwer et al. } \\
\text { (2007) }\end{array}$ \\
\hline Gabon & 1.58 & 1.58 & 0.00 & 3.15 & 3.15 & & 100.00 & 0.500 & 0.500 & 0.000 & Shares based on neighboring countries \\
\hline Gambia, The & 2.15 & 0.00 & 0.00 & 2.15 & 5.00 & & 46.53 & 1.000 & 0.000 & 0.000 & \\
\hline Georgia & 373.00 & 0.00 & 28.31 & 401.31 & 401.30 & & 31.42 & 0.929 & 0.000 & 0.071 & \\
\hline Germany & 10.70 & 500.00 & 5.00 & 515.70 & 515.70 & 540.00 & 45.49 & 0.021 & 0.970 & 0.010 & \\
\hline Ghana & 24.60 & 6.30 & 0.00 & 30.90 & 30.90 & & 90.32 & 0.796 & 0.204 & 0.000 & \\
\hline Greece & 77.75 & $1,088.50$ & 388.75 & $1,555.00$ & $1,555.00$ & & 82.32 & 0.050 & 0.700 & 0.250 & Shares based on neighboring countries \\
\hline Grenada & 0.00 & 0.03 & 0.19 & 0.22 & 0.22 & & & 0.000 & 0.132 & 0.868 & \\
\hline Guatemala & 198.60 & 94.43 & 19.08 & 312.11 & 337.50 & & 100.00 & 0.636 & 0.303 & 0.061 & \\
\hline Guinea & 19.93 & 0.30 & 0.16 & 20.39 & 20.39 & & 100.00 & 0.977 & 0.015 & 0.008 & \\
\hline Guinea-Bissau & 8.56 & 0.00 & 0.00 & 8.56 & 8.56 & & 100.00 & 1.000 & 0.000 & 0.000 & \\
\hline Guyana & 143.00 & 0.00 & 0.00 & 143.00 & 143.00 & & 89.16 & 1.000 & 0.000 & 0.000 & \\
\hline Haiti & 91.50 & 0.00 & 0.00 & 91.50 & 97.00 & & 71.50 & 1.000 & 0.000 & 0.000 & \\
\hline Holy See & 0.00 & 0.00 & 0.00 & 0.00 & 0.00 & & & 0.000 & 0.000 & 0.000 & \\
\hline Honduras & 73.21 & 0.00 & 0.00 & 73.21 & 89.70 & & 92.92 & 1.000 & 0.000 & 0.000 & \\
\hline Hungary & 18.90 & 118.00 & 4.00 & 140.90 & 140.90 & 220.00 & 62.19 & 0.134 & 0.837 & 0.028 & \\
\hline Iceland & 0.00 & 0.00 & 0.00 & 0.00 & 0.00 & & & 0.000 & 0.000 & 0.000 & \\
\hline India & $61,938.00$ & $1,446.00$ & 578.20 & $\begin{array}{l}63,962.2 \\
0\end{array}$ & $66,334.00$ & $60,900.00$ & 93.90 & 0.968 & 0.023 & 0.009 & \\
\hline Indonesia & $6,722.00$ & 0.00 & 0.00 & 6,722.00 & $6,722.00$ & & 100.00 & 1.000 & 0.000 & 0.000 & \\
\hline
\end{tabular}




\begin{tabular}{|c|c|c|c|c|c|c|c|c|c|c|c|}
\hline Iran & $7,970.00$ & 460.00 & 270.00 & $8,700.00$ & $8,700.00$ & $8,700.00$ & 77.41 & 0.916 & 0.053 & 0.031 & Based on ICID (2012) \\
\hline Iraq & $3,402.33$ & 96.59 & 26.09 & 3,525.01 & $3,525.00$ & & 54.89 & 0.965 & 0.027 & 0.007 & Based on Rohwer et al. (2007) \\
\hline Ireland & 0.00 & 0.50 & 0.60 & 1.10 & 1.10 & & 100.00 & 0.000 & 0.455 & 0.545 & Shares based on neighboring countries \\
\hline Israel & 0.00 & 60.00 & 168.80 & 228.80 & 225.00 & 231.00 & 80.67 & 0.000 & 0.262 & 0.738 & \\
\hline Italy & 2,399.00 & 981.20 & 570.60 & $3,950.80$ & $3,951.00$ & $2,670.00$ & 67.48 & 0.607 & 0.248 & 0.144 & \\
\hline Jamaica & 19.04 & 4.41 & 1.76 & 25.22 & 25.22 & & 100.00 & 0.755 & 0.175 & 0.070 & \\
\hline Japan & $2,010.00$ & 430.00 & 60.00 & $2,500.00$ & $2,500.00$ & $2,500.00$ & 92.86 & 0.804 & 0.172 & 0.024 & \\
\hline Jordan & 13.86 & 1.00 & 64.00 & 78.86 & 78.86 & & 95.11 & 0.176 & 0.013 & 0.812 & \\
\hline Kazakhstan & 713.00 & $1,400.00$ & 17.00 & $2,130.00$ & $1,200.00$ & $2,130.00$ & 98.50 & 0.335 & 0.657 & 0.008 & Based on ICID (2012) \\
\hline Kenya & 39.22 & 61.99 & 2.00 & 103.21 & 103.20 & & 94.19 & 0.380 & 0.601 & 0.019 & \\
\hline Kiribati & 0.00 & 0.00 & 0.00 & 0.00 & 0.00 & & & 0.000 & 0.000 & 0.000 & \\
\hline Kuwait & 3.02 & 0.60 & 1.15 & 4.77 & 10.14 & & 69.53 & 0.633 & 0.126 & 0.241 & \\
\hline Kyrgyzstan & $1,021.00$ & 0.40 & 0.00 & $1,021.40$ & $1,021.00$ & & 100.00 & 1.000 & 0.000 & 0.000 & \\
\hline Laos & 155.40 & 0.00 & 0.00 & 155.40 & 310.00 & & 87.32 & 1.000 & 0.000 & 0.000 & \\
\hline Latvia & 0.00 & 0.00 & 0.00 & 0.00 & 0.83 & & 74.70 & 0.000 & 0.000 & 0.000 & \\
\hline Lebanon & 66.13 & 29.04 & 8.84 & 104.01 & 104.00 & & 86.54 & 0.636 & 0.279 & 0.085 & \\
\hline Lesotho & 1.32 & 1.00 & 0.32 & 2.64 & 2.64 & & 2.54 & 0.500 & 0.379 & 0.121 & Shares based on neighboring countries \\
\hline Liberia & 0.10 & 0.00 & 0.00 & 0.10 & 0.10 & & 100.00 & 1.000 & 0.000 & 0.000 & Shares based on neighboring countries \\
\hline Libya & 423.00 & 23.50 & 23.50 & 470.00 & 470.00 & & 67.23 & 0.900 & 0.050 & 0.050 & Shares based on neighboring countries \\
\hline Liechtenstein & 0.00 & 0.00 & 0.00 & 0.00 & 0.00 & & & 0.000 & 0.000 & 0.000 & \\
\hline Lithuania & 0.00 & 9.25 & 0.00 & 9.25 & 1.34 & 4.40 & 74.63 & 0.000 & 1.000 & 0.000 & \\
\hline Luxembourg & 0.00 & 0.00 & 0.00 & 0.00 & 0.00 & & & 0.000 & 0.000 & 0.000 & \\
\hline Madagascar & $1,084.00$ & 2.40 & 0.00 & $1,086.40$ & $1,086.00$ & & 50.64 & 0.998 & 0.002 & 0.000 & \\
\hline Malawi & 6.36 & 43.19 & 5.45 & 55.00 & 73.50 & 55.00 & 98.18 & 0.116 & 0.785 & 0.099 & \\
\hline Malaysia & 373.00 & 2.00 & 5.00 & 380.00 & 380.00 & 380.00 & 76.37 & 0.982 & 0.005 & 0.013 & \\
\hline Maldives & 0.00 & 0.00 & 0.00 & 0.00 & 0.00 & & & 0.000 & 0.000 & 0.000 & \\
\hline Mali & 166.90 & 0.03 & 0.14 & 167.07 & 167.10 & & 83.72 & 0.999 & 0.000 & 0.001 & \\
\hline Malta & 0.11 & 0.15 & 0.50 & 0.76 & 3.20 & & 87.81 & 0.148 & 0.197 & 0.655 & \\
\hline Marshall Islands & 0.00 & 0.00 & 0.00 & 0.00 & 0.00 & & & 0.000 & 0.000 & 0.000 & \\
\hline Mauritania & 43.70 & 1.31 & 0.00 & 45.01 & 45.01 & & 50.74 & 0.971 & 0.029 & 0.000 & Shares based on neighboring countries \\
\hline
\end{tabular}




\begin{tabular}{|c|c|c|c|c|c|c|c|c|c|c|c|}
\hline Mauritius & 2.37 & 17.03 & 1.82 & 21.22 & 21.22 & & 98.02 & 0.112 & 0.802 & 0.086 & \\
\hline Mexico & $5,168.00$ & 310.80 & 143.10 & $5,621.90$ & $6,460.00$ & $6,200.00$ & 86.06 & 0.919 & 0.055 & 0.025 & \\
\hline $\begin{array}{l}\text { Federated States of } \\
\text { Micronesia }\end{array}$ & 0.00 & 0.00 & 0.00 & 0.00 & 0.00 & & & 0.000 & 0.000 & 0.000 & \\
\hline Monaco & 0.00 & 0.00 & 0.00 & 0.00 & 0.00 & & & 0.000 & 0.000 & 0.000 & \\
\hline Mongolia & 13.90 & 43.40 & 0.00 & 57.30 & 57.30 & & 61.08 & 0.243 & 0.757 & 0.000 & \\
\hline Montenegro & 0.00 & 0.90 & 1.50 & 2.41 & 2.41 & & 100.00 & 0.002 & 0.375 & 0.624 & \\
\hline Morocco & $1,209.00$ & 151.70 & 97.97 & $1,458.67$ & $1,459.00$ & $1,650.00$ & 99.04 & 0.829 & 0.104 & 0.067 & \\
\hline Mozambique & 131.04 & 156.00 & 24.96 & 312.00 & 118.10 & & 33.92 & 0.420 & 0.500 & 0.080 & Based on Rohwer et al. (2007) \\
\hline Myanmar (Burma) & $2,083.00$ & 0.00 & 0.00 & $2,083.00$ & $2,083.00$ & & 100.00 & 1.000 & 0.000 & 0.000 & \\
\hline Namibia & 2.95 & 3.28 & 1.35 & 7.57 & 7.57 & & 100.00 & 0.390 & 0.433 & 0.178 & \\
\hline Nauru & 0.00 & 0.00 & 0.00 & 0.00 & 0.00 & & & 0.000 & 0.000 & 0.000 & \\
\hline Nepal & $1,134.00$ & 0.00 & 0.00 & $1,134.00$ & $1,168.00$ & & & 1.000 & 0.000 & 0.000 & \\
\hline Netherlands & 152.40 & 152.40 & 152.40 & 457.20 & 457.20 & & 44.25 & 0.333 & 0.333 & 0.333 & Based on Rohwer et al. (2007) \\
\hline New Zealand & 110.90 & 491.40 & 41.66 & 643.96 & 619.30 & & 82.19 & 0.172 & 0.763 & 0.065 & \\
\hline Nicaragua & 61.37 & 0.00 & 0.10 & 61.47 & 199.10 & & 72.38 & 0.998 & 0.000 & 0.002 & \\
\hline Niger & 13.74 & 0.00 & 0.00 & 13.74 & 13.74 & & 77.00 & 1.000 & 0.000 & 0.000 & \\
\hline Nigeria & 238.10 & 0.05 & 0.00 & 238.15 & 238.20 & & 91.86 & 1.000 & 0.000 & 0.000 & \\
\hline Niue & & & & 0.00 & & & & 0.000 & 0.000 & 0.000 & \\
\hline Norway & 61.35 & 0.01 & 0.00 & 61.36 & 114.90 & & 47.84 & 1.000 & 0.000 & 0.000 & \\
\hline West Bank & & 12.00 & 12.00 & 24.00 & 24.00 & & 100.10 & 0.000 & 0.500 & 0.500 & Shares based on neighboring countries \\
\hline Oman & 46.66 & 6.65 & 5.54 & 58.85 & 58.85 & & 100.00 & 0.793 & 0.113 & 0.094 & \\
\hline Pakistan & $19,270.00$ & 0.00 & 0.00 & $\begin{array}{l}19,270.0 \\
0\end{array}$ & $19,270.00$ & & 100.00 & 1.000 & 0.000 & 0.000 & \\
\hline Palau & & & & 0.00 & & & & 0.000 & 0.000 & 0.000 & \\
\hline Panama & 23.90 & 3.74 & 4.50 & 32.14 & 32.14 & & 100.00 & 0.744 & 0.116 & 0.140 & \\
\hline Papua New Guinea & 45.73 & 14.77 & 1.05 & 61.55 & 62.55 & & & 0.743 & 0.240 & 0.017 & Based on Rohwer et al. (2007) \\
\hline Paraguay & 108.96 & 13.62 & 13.62 & 136.20 & 136.20 & & 100.00 & 0.800 & 0.100 & 0.100 & Shares based on neighboring countries \\
\hline Peru & $1,697.00$ & 21.74 & 10.63 & $1,729.37$ & $1,729.00$ & & 64.14 & 0.981 & 0.013 & 0.006 & \\
\hline Philippines & $1,864.00$ & 4.50 & 10.92 & $1,879.42$ & $1,879.00$ & $1,520.00$ & 100.00 & 0.992 & 0.002 & 0.006 & \\
\hline Poland & 102.70 & 5.00 & 8.00 & 115.70 & 115.70 & 100.00 & 62.28 & 0.888 & 0.043 & 0.069 & \\
\hline
\end{tabular}




\begin{tabular}{|c|c|c|c|c|c|c|c|c|c|c|c|}
\hline Portugal & 585.00 & 40.00 & 25.00 & 650.00 & 583.70 & 630.00 & 72.21 & 0.900 & 0.062 & 0.038 & $\begin{array}{l}\text { Complemented with ICID (2012) and } \\
\text { Rohwer et al. (2007) }\end{array}$ \\
\hline Puerto Rico & $1,460.00$ & 95.38 & 23.84 & $1,579.22$ & 22.04 & & 71.60 & 0.925 & 0.060 & 0.015 & Based on Rohwer et al. (2007) \\
\hline Qatar & 9.71 & 1.81 & 1.42 & 12.94 & 12.94 & & 46.85 & 0.750 & 0.140 & 0.109 & \\
\hline South Korea & 888.80 & 0.00 & 0.00 & 888.80 & 880.40 & $1,010.00$ & & 1.000 & 0.000 & 0.000 & \\
\hline Moldova & 68.00 & 145.00 & 15.00 & 228.00 & 228.30 & & 72.12 & 0.298 & 0.636 & 0.066 & \\
\hline Romania & 420.00 & 448.00 & 4.00 & 872.00 & 615.30 & $1,500.00$ & 28.18 & 0.482 & 0.514 & 0.005 & \\
\hline Russia & $1,953.00$ & $2,500.00$ & 47.00 & $4,500.00$ & $2,375.00$ & $4,500.00$ & & 0.434 & 0.556 & 0.010 & Based on ICID (2012) \\
\hline Rwanda & 3.50 & & & 3.50 & 4.63 & & 43.24 & 1.000 & 0.000 & 0.000 & \\
\hline Saint Kitts and Nevis & 0.00 & 0.00 & 0.02 & 0.02 & 0.02 & & & 0.000 & 0.000 & 1.000 & \\
\hline Saint Lucia & & & & 0.00 & 3.00 & & & 0.000 & 0.000 & 0.000 & \\
\hline $\begin{array}{l}\text { Saint Vincent and the } \\
\text { Grenadines }\end{array}$ & & & & 0.00 & & & & 0.000 & 0.000 & 0.000 & \\
\hline American Samoa & & & & 0.00 & & & & 0.000 & 0.000 & 0.000 & \\
\hline San Marino & & & & 0.00 & & & & 0.000 & 0.000 & 0.000 & \\
\hline $\begin{array}{l}\text { Sao Tome and } \\
\text { Principe }\end{array}$ & & & & 0.00 & 9.70 & & & 0.000 & 0.000 & 0.000 & \\
\hline Saudi Arabia & 706.00 & 716.00 & 198.00 & $1,620.00$ & $1,620.00$ & $1,620.00$ & 100.00 & 0.436 & 0.442 & 0.122 & \\
\hline Senegal & 102.20 & & 0.40 & 102.60 & 102.20 & & 67.51 & 0.996 & 0.000 & 0.004 & \\
\hline Serbia & 64.37 & 22.99 & 4.59 & 91.95 & 91.96 & & 37.16 & 0.700 & 0.250 & 0.050 & Shares based on neighboring countries \\
\hline Seychelles & 0.02 & 0.04 & 0.20 & 0.26 & 0.26 & & 76.92 & 0.077 & 0.154 & 0.769 & \\
\hline Sierra Leone & 1.00 & 0.00 & 0.00 & 1.00 & 1.00 & & 100.00 & 1.000 & 0.000 & 0.000 & \\
\hline Singapore & & & & 0.00 & & & & 0.000 & 0.000 & 0.000 & \\
\hline Slovakia & 0.35 & 310.00 & 2.65 & 313.00 & 172.00 & 313.00 & 22.73 & 0.001 & 0.990 & 0.008 & Based on ICID (2012) \\
\hline Slovenia & 0.00 & 5.27 & 2.34 & 7.60 & 7.60 & 7.30 & 46.04 & 0.000 & 0.693 & 0.307 & \\
\hline Solomon Islands & & & & 0.00 & & & & 0.000 & 0.000 & 0.000 & \\
\hline Somalia & 50.00 & & & 50.00 & 50.00 & & 100.00 & 1.000 & 0.000 & 0.000 & \\
\hline South Africa & 385.00 & 920.00 & 365.00 & $1,670.00$ & $1,670.00$ & $1,670.00$ & 95.87 & 0.231 & 0.551 & 0.219 & \\
\hline South Sudan & & & & 0.00 & & & & 0.000 & 0.000 & 0.000 & \\
\hline Spain & $1,029.00$ & 783.00 & $1,658.00$ & $3,470.00$ & $3,470.00$ & $3,470.00$ & 89.14 & 0.297 & 0.226 & 0.478 & \\
\hline Sri Lanka & 570.00 & 0.00 & 0.00 & 570.00 & 570.00 & & 81.14 & 1.000 & 0.000 & 0.000 & \\
\hline
\end{tabular}




\begin{tabular}{|c|c|c|c|c|c|c|c|c|c|c|c|}
\hline Sudan & $1,758.00$ & & & $1,758.00$ & $1,758.00$ & & & 1.000 & 0.000 & 0.000 & \\
\hline Suriname & 50.32 & 1.10 & 0.00 & 51.42 & 57.00 & & 100.00 & 0.979 & 0.021 & 0.000 & \\
\hline Swaziland & 25.89 & 20.91 & 3.05 & 49.85 & 49.85 & & 89.95 & 0.519 & 0.419 & 0.061 & \\
\hline Sweden & 0.00 & 159.70 & 0.00 & 159.70 & 159.70 & & 33.92 & 0.000 & 1.000 & 0.000 & \\
\hline Switzerland & 0.83 & 59.17 & 1.00 & 61.00 & 61.00 & & 59.31 & 0.014 & 0.970 & 0.016 & Shares based on neighboring countries \\
\hline Syria & $1,043.00$ & 187.10 & 110.90 & $1,341.00$ & $1,341.00$ & $1,280.00$ & 95.50 & 0.778 & 0.140 & 0.083 & \\
\hline Tajikistan & 742.10 & 0.00 & 0.00 & 742.10 & 742.10 & & 90.88 & 1.000 & 0.000 & 0.000 & \\
\hline Thailand & $6,415.00$ & & & $6,415.00$ & $6,415.00$ & & 78.88 & 1.000 & 0.000 & 0.000 & \\
\hline Macedonia & 49.00 & 5.00 & 1.00 & 55.00 & 127.80 & & 62.32 & 0.891 & 0.091 & 0.018 & Based on ICID (2012) \\
\hline Timor-Leste & & & & 0.00 & 34.65 & & 83.43 & 0.000 & 0.000 & 0.000 & \\
\hline Togo & 2.30 & 0.55 & 0.01 & 2.86 & 2.30 & & 47.39 & 0.804 & 0.192 & 0.003 & \\
\hline Tokelau & & & & 0.00 & & & & 0.000 & 0.000 & 0.000 & \\
\hline Tonga & & & & 0.00 & & & & 0.000 & 0.000 & 0.000 & \\
\hline Trinidad and Tobago & 2.89 & 0.71 & 0.12 & 3.71 & 3.60 & & 85.00 & 0.778 & 0.190 & 0.032 & \\
\hline Tunisia & 215.00 & 90.00 & 62.00 & 367.00 & 367.00 & & 100.00 & 0.586 & 0.245 & 0.169 & \\
\hline Turkey & $4,690.00$ & 500.00 & 150.00 & $5,340.00$ & $5,340.00$ & $5,340.00$ & 84.63 & 0.878 & 0.094 & 0.028 & \\
\hline Turkmenistan & $1,991.00$ & 0.00 & 0.00 & $1,991.00$ & $1,991.00$ & & 100.00 & 1.000 & 0.000 & 0.000 & \\
\hline Tuvalu & & & & 0.00 & & & & 0.000 & 0.000 & 0.000 & \\
\hline Uganda & 5.35 & 0.23 & & 5.58 & 12.08 & & 100.00 & 0.959 & 0.041 & 0.000 & \\
\hline Ukraine & 525.00 & $2,080.00$ & 0.00 & $2,605.00$ & $2,175.00$ & $2,180.00$ & & 0.202 & 0.798 & 0.000 & \\
\hline $\begin{array}{l}\text { United Arab } \\
\text { Emirates }\end{array}$ & 27.10 & 4.00 & 195.50 & 226.60 & 92.00 & & 82.61 & 0.120 & 0.018 & 0.863 & \\
\hline United Kingdom & 117.00 & 105.00 & 6.00 & 228.00 & 228.00 & 110.00 & 60.61 & 0.513 & 0.461 & 0.026 & \\
\hline $\begin{array}{l}\text { Tanzania, United } \\
\text { Republic of }\end{array}$ & 184.30 & & & 184.30 & 184.30 & & 100.00 & 1.000 & 0.000 & 0.000 & \\
\hline United States & $12,696.00$ & $12,333.00$ & $1,615.00$ & $\begin{array}{l}26,644.0 \\
0\end{array}$ & $26,644.00$ & $24,700.00$ & 85.97 & 0.477 & 0.463 & 0.061 & \\
\hline Uruguay & 200.00 & 18.00 & 20.00 & 238.00 & 238.00 & & 100.00 & 0.840 & 0.076 & 0.084 & \\
\hline Uzbekistan & $4,276.00$ & 0.00 & 4.51 & $4,280.51$ & $4,198.00$ & $4,233.00$ & 88.14 & 0.999 & 0.000 & 0.001 & \\
\hline Vanuatu & & & & 0.00 & & & & 0.000 & 0.000 & 0.000 & \\
\hline Venezuela & 735.50 & 275.50 & 44.30 & $1,055.30$ & $1,055.00$ & & 92.78 & 0.697 & 0.261 & 0.042 & \\
\hline
\end{tabular}




\begin{tabular}{|l|l|l|l|l|l|l|l|l|l|l|l|}
\hline Vietnam & $4,584.00$ & 1.10 & 0.00 & $4,585.10$ & $4,585.00$ & & 100.00 & 1.000 & 0.000 & 0.000 & \\
\hline Yemen & 453.80 & 0.35 & 0.49 & 454.64 & 454.30 & & 100.00 & 0.998 & 0.001 & 0.001 & \\
\hline Zambia & 32.19 & 17.57 & 5.63 & 55.39 & 55.39 & & 100.00 & 0.581 & 0.317 & 0.102 & \\
\hline Zimbabwe & 46.85 & 112.80 & 13.88 & 173.53 & 173.50 & & 71.41 & 0.270 & 0.650 & 0.080 & \\
\hline
\end{tabular}

${ }^{\mathrm{a}}$ in 1000 ha, ${ }^{\mathrm{b}}$ calculated from the extent of areas with surface, sprinkler and drip irrigation (column 1-3) 


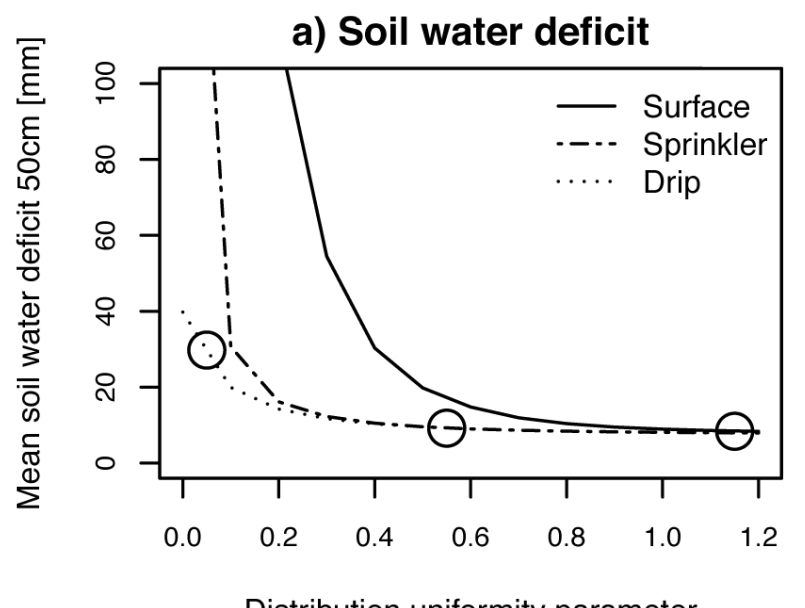

Distribution uniformity parameter

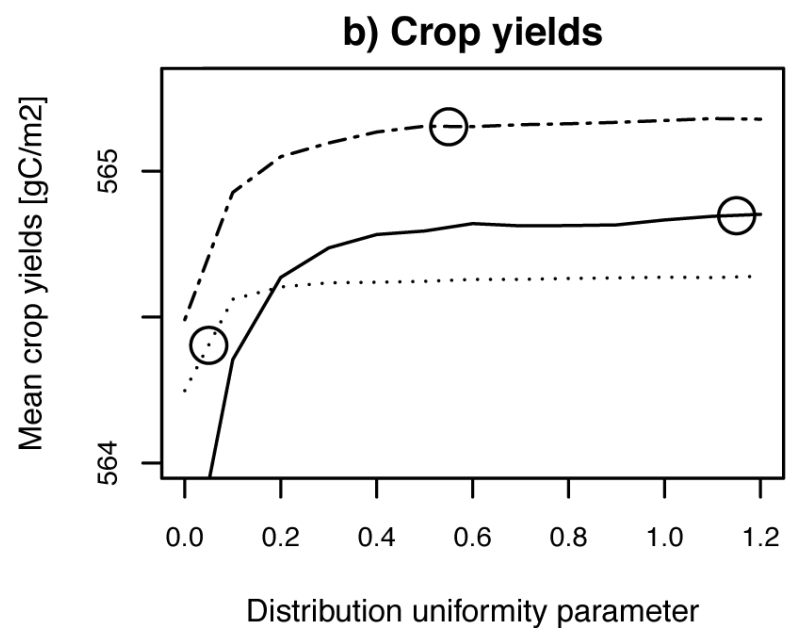

Figure S1: Parameter sensitivity analysis for the distribution uniformity scalar ( $d u)$. Panel (a) shows the dependency on annually cumulated soil water deficit in the upper $50 \mathrm{~cm}$ (irrigation depth) that could not be fulfilled by irrigation, as mean over irrigated cells. Panel (b) shows the dependency on mean annual crop yield. Simulations are based on All-Surf, All-Sprinkler, and AllDrip scenarios (see main text, 2.5) assuming unrestricted water availability for irrigation. Employed parameter estimates are indicated. Surface and sprinkler systems are parameterized to meet net irrigation requirement (field capacity in upper $50 \mathrm{~cm}$ ) and thus yield production is at its potential. Drip systems are parameterized to represent a modest form of deficit irrigation, not to maximize yields but to save water.

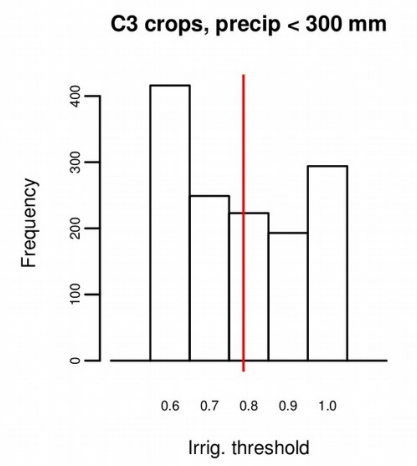

C4 crops, precip $<300 \mathrm{~mm}$

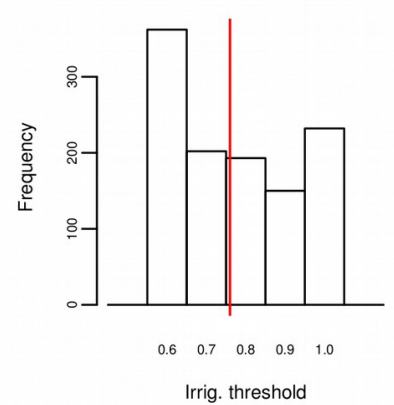

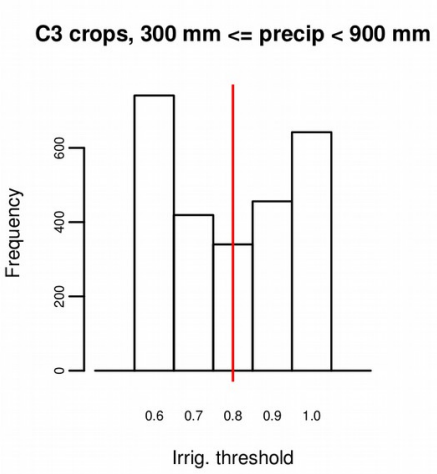

C4 crops, $300 \mathrm{~mm}<=$ precip $<900 \mathrm{~mm}$

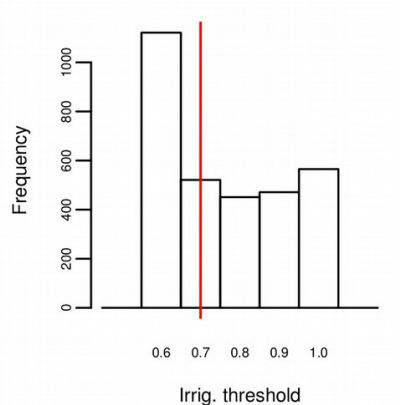

Irrig. threshold

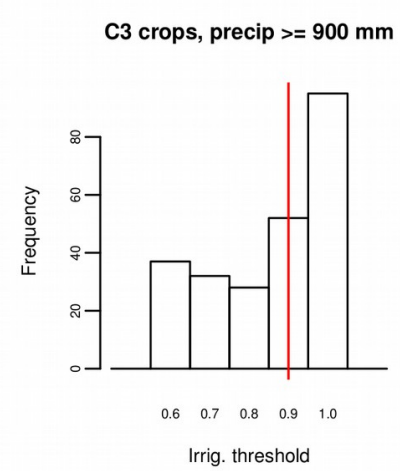

C4 crops, precip >= $900 \mathrm{~mm}$

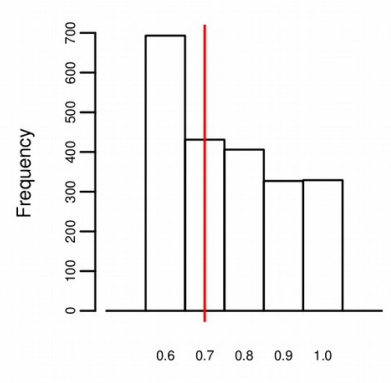

Irrig. threshold

- Median

Figure S2: Sensitivity analysis for the irrigation threshold (it) parameter. Stacks represent the number of cells that achieve their maximum harvest at the respective it and the red line is the median across all cells. The two rows represent C3 and C4 crops, respectively, and the columns represent the indicated precipitation regimes. 
Return-flow
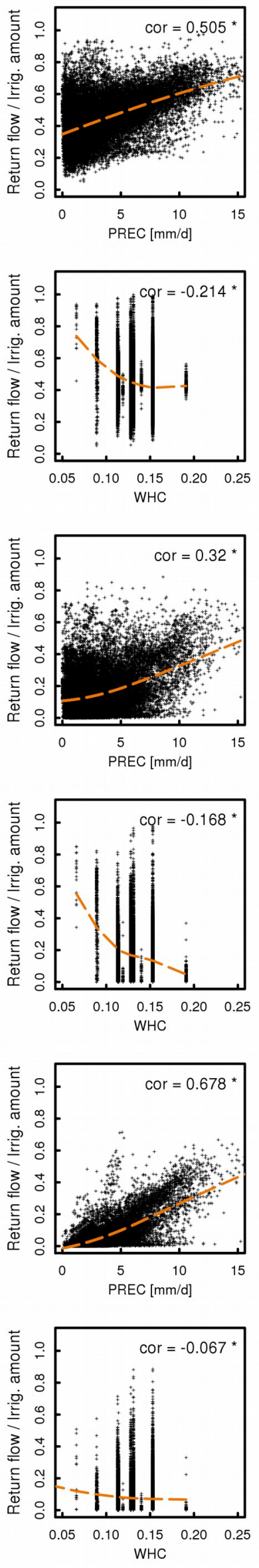

Soll evap.
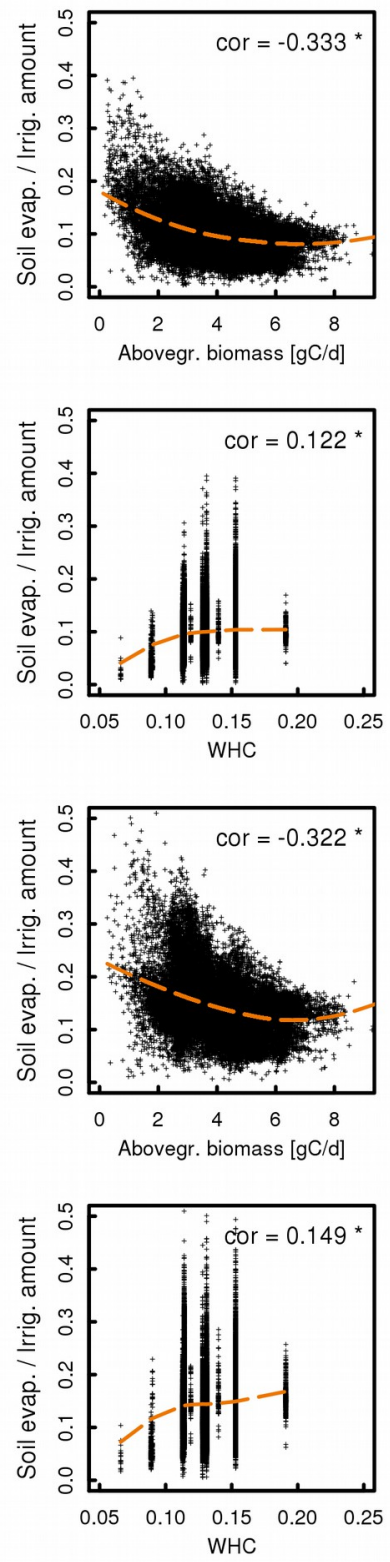
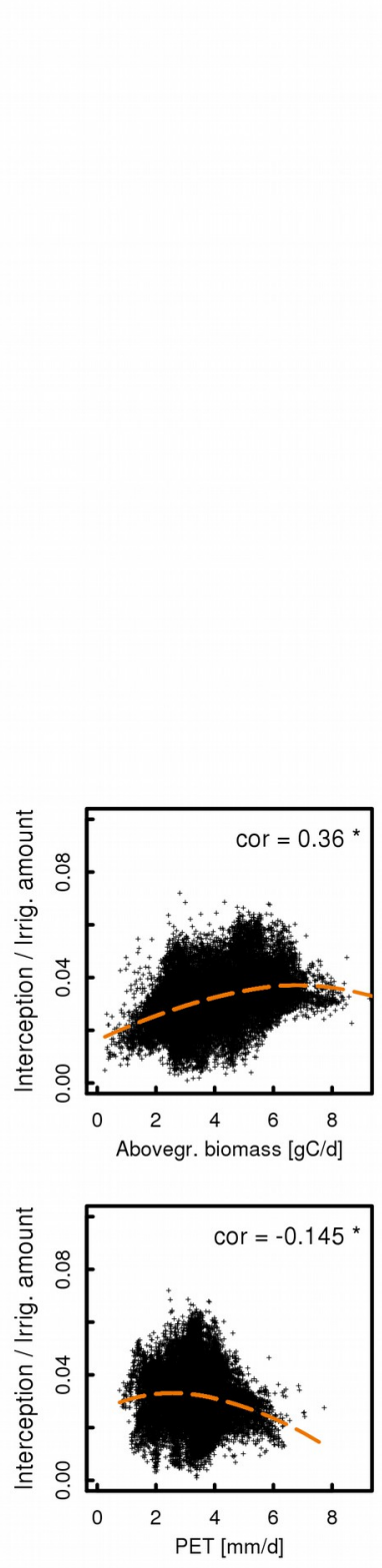

Interception

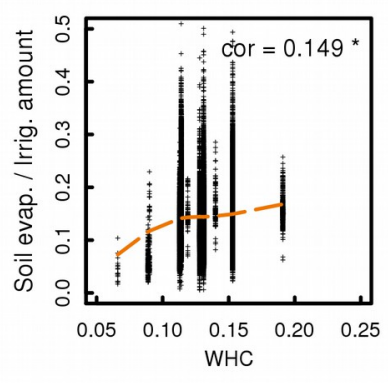


Figure S3: Dependencies of non-beneficial irrigation water fluxes on its main biophysical driving factors (3 columns: return flow, soil evaporation, interception). Double rows represent the irrigation system: surface, sprinkler, and drip. Data is based on 12 crop CFTs from the All-Surface, AllSprinkler and All-Drip scenario, respectively and country-scale management intensities are harmonized (calibration factors are set so as to represent systems with optimal management). Dashed lines indicate polynomial bias curve, "cor" refers to Spearman's correlation coefficient (*, if p-value $<10^{-9}$ ). PREC: precipitation, WHC: water holding capacity, PET: potential evapotranspiration.

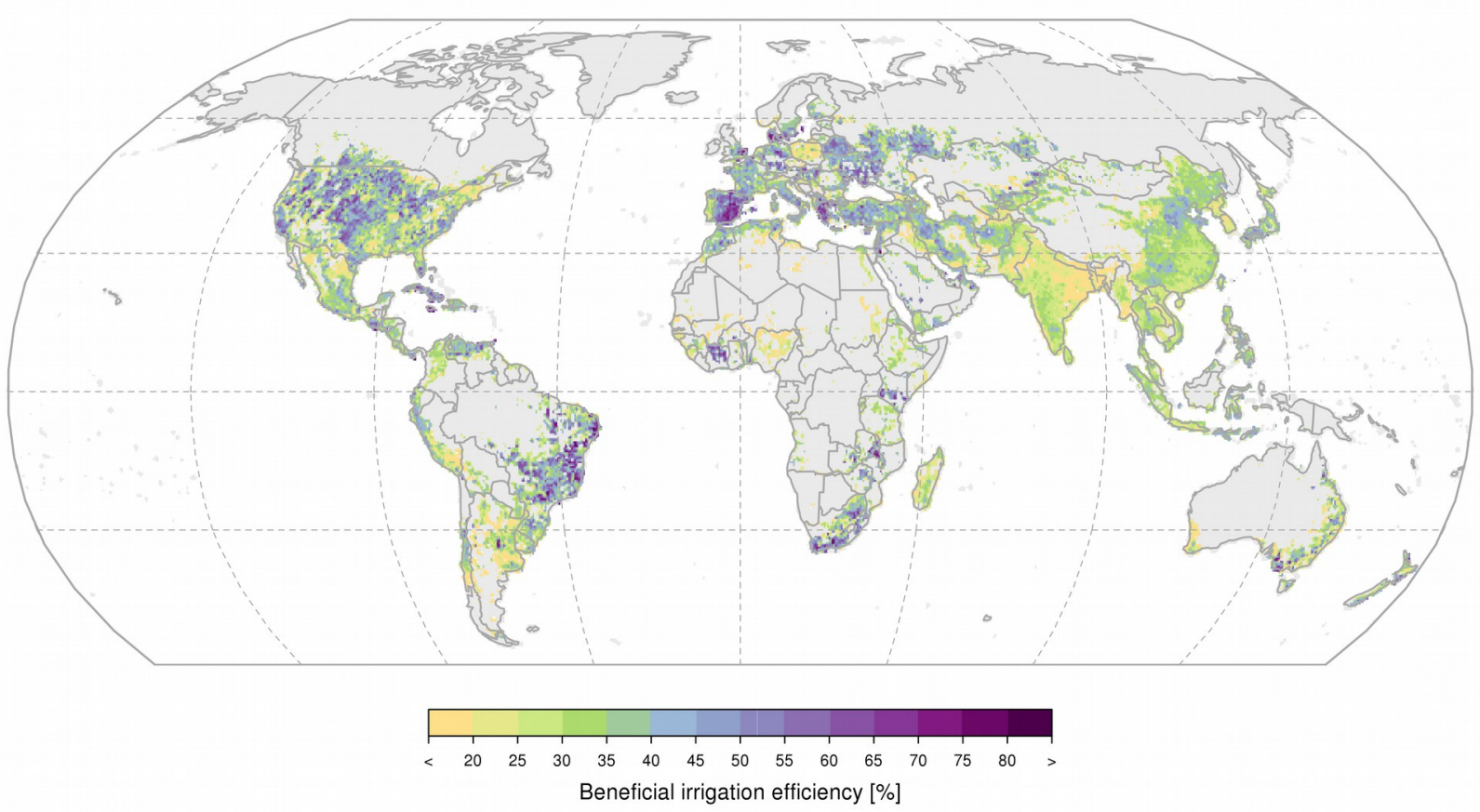

Figure S4: Global patterns of beneficial irrigation efficiency $\left(\mathrm{E}_{\mathrm{b}}\right.$, ratio of transpired and diverted water) as area-weighted mean over CFTs (inclusive "others" and pastures) and based on the system distribution in Figure 3. Gridded data for this figure are provided as Supplement for other studies.

\section{References}

Brouwer, C., Prins, K., Kay, M., \& Heibloem, M. (1988). Irrigation Water Management : Irrigation Methods. Training manual no. 5.

FAO. (2014). AQUASTAT database - Food and Agriculture Organization of the United Nations (FAO). Retrieved from http://www.fao.org/nr/water/aquastat/data/query/index.html?lang=en

Fischer, G., Nachtergaele, F., Prieler, S., Teixeira, E., Tóth, G., van Velthuizen, H., ... Wiberg, D. (2012). Global Agro-ecological Zones (GAEZ v3.0). IIASA, Laxenburg, Austria and FAO, Rome, Italy.

ICID. (2012). Sprinkler and micro irrigated area - Commission on Irrigation and Drainage (ICID).

Portmann, F. T., Siebert, S., \& Döll, P. (2010). MIRCA2000—Global monthly irrigated and rainfed crop areas around the year 2000: A new high-resolution data set for agricultural and 
hydrological modeling. Global Biogeochemical Cycles, 24(1). doi:10.1029/2008GB003435

Rohwer, J., Gerten, D., \& Lucht, W. (2007). Development of functional irrigation types for improved global crop modelling - PIK report No. 104. Potsdam, Germany.

Sauer, T., Havlík, P., Schneider, U. a., Schmid, E., Kindermann, G., \& Obersteiner, M. (2010). Agriculture and resource availability in a changing world: The role of irrigation. Water Resources Research, 46(6), n/a-n/a. doi:10.1029/2009WR007729

Siebert, S., Burke, J., Faures, J. M., Frenken, K., Hoogeveen, J., Döll, P., \& Portmann, F. T. (2010). Groundwater use for irrigation - a global inventory. Hydrology and Earth System Sciences, 14(10), 1863-1880. doi:10.5194/hess-14-1863-2010

Siebert, S., Döll, P., Feick, S., Hoogeveen, J., \& Frenken, K. (2007). Global Map of Irrigation Areas Version 4.0.1 [CD-Rom], FAO Land and Water Digital Media Ser. 34. Rome, FAO.

Siebert, S., Döll, P., Hoogeveen, J., Faures, J.-M., Frenken, K., \& Feick, S. (2005). Development and validation of the global map of irrigation areas. Hydrology and Earth System Sciences. doi:10.5194/hessd-2-1299-2005

Siebert, S., Kummu, M., Porkka, M., Döll, P., Ramankutty, N., \& Scanlon, B. R. (2014). A global dataset of the extent of irrigated land from 1900 to 2005. Hydrology and Earth System Sciences Discussions, (11), 13207-13258. doi:10.13019/M2MW2G

Siebert, S., Kummu, M., Porkka, M., Döll, P., Ramankutty, N., \& Scanlon, B. R. (2015). A global data set of the extent of irrigated land from 1900 to 2005. Hydrology and Earth System Sciences, (19), 1521-1545. doi:10.13019/M20599 